\title{
Pulmonary Involvement in the Antisynthetase Syndrome: A Comparative Cross-sectional Study
}

\author{
Helena Andersson, Trond Mogens Aaløkken, Anne Günther, Georg Karl Mynarek, \\ Torhild Garen, May Brit Lund, and Øyvind Molberg
}

ABSTRACT. Objective. Interstitial lung disease (ILD) is a major component of the antisynthetase syndrome, but quantitative data on longterm pulmonary outcome in antisynthetase syndrome are limited. In this study, the main aims were to compare pulmonary function tests (PFT) and the 6-min walking distance (6MWD) between patients with antisynthetase syndrome and healthy sex- and age-matched controls, to evaluate the extent of ILD by lung high-resolution computed tomography (HRCT), and to assess correlations between PFT measures and ILD extent.

Methods. Concurrent PFT and 6MWD were performed in 68 patients with antisynthetase syndrome and their individually matched controls. Additionally, in the patients, the extent of ILD was determined in 10 HRCT sections, expressed as percentage of total lung volumes.

Results. Median disease duration in the antisynthetase syndrome cohort was 71 months. Compared with the matched controls, the patients with antisynthetase syndrome had mean $28 \%, 27 \%$, and $53 \%$ lower absolute values of forced vital capacity (FVC), forced expiratory volume in $1 \mathrm{~s}$, and DLCO $(\mathrm{p}<0.001)$. Mean difference in 6MWD between patients and controls was $116 \mathrm{~m}(\mathrm{p}<0.001)$. Median extent of ILD by HRCT was $20 \%$ (range $0-73$ ) and correlated with FVC and DLCO. Pulmonary outcome did not differ between Jo1 and non-Jo1 subsets.

Conclusion. To our knowledge, this study is the first to demonstrate a highly significant difference in PFT between patients with antisynthetase syndrome with 6 years of followup and healthy controls. DLCO displayed the highest difference with mean 53\% lower value in the patients. FVC and DLCO correlated significantly with ILD extent, indicating these variables as appropriate outcome measures in antisynthetase syndrome-associated ILD. (First Release April 1 2016; J Rheumatol 2016; 43:1107-13; doi:10.3899/jrheum.151067)

Key Indexing Terms:

ANTISYNTHETASE ANTIBODY PULMONARY FUNCTION TEST

\section{INTERSTITIAL LUNG DISEASE ANTISYNTHETASE SYNDROME MYOSITIS}

The antisynthetase syndrome was first described in patients with polymyositis (PM) or dermatomyositis (DM) ${ }^{1}$, but has also been identified in patients without clinical myositis ${ }^{2,3}$. Antisynthetase syndrome is defined by the presence of an antiaminoacyl-t-RNA synthetase (aaRS) antibody and distinct clinical features including arthritis, Raynaud phenomenon, fever, mechanic hands, myositis, and interstitial lung

From the Institute of Clinical Medicine, and Department of Rheumatology, and Department of Radiology, and Department of Respiratory Medicine, Oslo University Hospital, Oslo, Norway.

Supported by grants from the Norwegian Women's Public Health Association and the Norwegian Rheumatology Foundation.

H. Andersson, MD, Institute of Clinical Medicine, and Department of Rheumatology, Oslo University Hospital; T.M. Aalфkken, MD, PhD,

Department of Radiology, Oslo University Hospital; A. Günther, MD,

Department of Radiology, Oslo University Hospital; G.K. Mynarek, MD,

Department of Radiology, Oslo University Hospital; T. Garen, MSc,

Department of Rheumatology, Oslo University Hospital; M.B. Lund, MD,

PhD, Department of Respiratory Medicine, Oslo University Hospital;

$\emptyset$. Molberg, Professor, Institute of Clinical Medicine, and Department of

Rheumatology, Oslo University Hospital.

Address correspondence to Dr. H. Andersson, Department of

Rheumatology, Oslo University Hospital, Pb 4950 Nydalen, 0424 Oslo,

Norway.E-mail: helena.andersson@medisin.uio.no

Accepted for publication February 25, 2016. disease (ILD). Eight anti-aaRS antibodies have been identified, the most common being anti-Jo1, present in $15 \%-30 \%$ of all patients with $\mathrm{PM} / \mathrm{DM}^{4}$. Previous studies have described ILD in $67 \%-100 \%$ of patients with antisynthetase syndrome, and it appears that the accumulated frequency of ILD in antisynthetase syndrome increases over time ${ }^{5}$. Risk factor analyses indicate that ILD is a major determinant of morbidity and mortality in the syndrome $6,7,8,9$. Further, severity of dyspnea and isolated ILD at diagnosis are 2 of the factors found to be associated with poor outcome in antisynthetase syndrome; the others are older age, diagnostic delay, and positive anti-SSA antibodies ${ }^{10,11,12,13,14}$.

Even though available evidence suggests that ILD is a major component of antisynthetase syndrome, only a few studies have assessed pulmonary outcome quantitatively. Johnson, et al compared pulmonary outcome between 41 patients with antisynthetase syndrome and 36 patients with anti-aaRS-negative myositis and ILD. With a mean followup of 28 months, they found a significantly lower forced vital capacity (FVC) and higher total computed tomography (CT) score in Jo1-positive patients compared with anti-aaRS-negative patients ${ }^{15}$. Some studies indicate that patients with

Personal non-commercial use only. The Journal of Rheumatology Copyright (c) 2016. All rights reserved. 
antisynthetase syndrome with non-Jo1 antibodies (i.e., non-Jo1 antisynthetase syndrome) have a poorer pulmonary outcome compared to the Jo1-positive patients ${ }^{16,17}$, but in a study from France, there was no difference in the pulmonary function test (PFT) results between Jo1 and non-Jo1 patients with antisynthetase syndrome ${ }^{18}$. The latter study did, however, show that the non-Jo1 patients had lower pulmonary remission rates and higher $\mathrm{CT}$ fibrosis scores after $>18$ months of followup than did the anti-Jo1-positive patients ${ }^{18}$. Recently, another group from France assessed total ILD extent by CT in 26 patients with antisynthetase syndrome with $>12$ months of followup, and found no difference between anti-Jo1 and PL-12-positive patients ${ }^{19}$.

Altogether, there appears to be substantial knowledge gaps on longterm pulmonary outcome in antisynthetase syndrome. To our knowledge, there is no study comparing pulmonary function between patients with antisynthetase syndrome and healthy, individually matched control subjects. Further, little is known about the correlations between PFT and the ILD extent by high-resolution CT (HRCT). Hence, the aims of our study were to (1) assess PFT and the 6-min walking distance (6MWD) in a cohort of largely unselected patients with antisynthetase syndrome and matched controls, and retrospectively evaluate $\mathrm{PFT}$ changes from baseline to study inclusion in the antisynthetase syndrome cohort; (2) evaluate correlations between PFT variables, especially FVC, and the extent of ILD by concurrent HRCT examinations; extent of ILD and FVC were selected because these 2 variables were suggested as core domains in clinical trials with connective tissue disease (CTD)-associated ILD ${ }^{20}$; and (3) evaluate any possible differences in pulmonary outcome between the Jo1 and non-Jo1 patients.

\section{MATERIALS AND METHODS}

Study population. The Oslo University Hospital (OUH) antisynthetase syndrome cohort is a single referral center cohort that by 2011 included 96 patients with positive aaRS antibodies, ILD defined by the American Thoracic Society (ATS) criteria $^{21}$, and/or probable or definite PM/DM according to the Bohan and Peter criteria ${ }^{22}$. The OUH antisynthetase syndrome cohort is largely unselected because it includes all the patients with antisynthetase syndrome identified in the population-based myositis cohort from Southeast Norway ${ }^{23}$. Our current study was performed between September 2011 and June 2014, including 68 of the 70 patients with antisynthetase syndrome who were alive by September 2011 (2 patients declined participation). Disease duration was defined as time from diagnosis to date of examination. Sex- and aged-matched healthy controls were randomly collected in a 1:1 proportion from the National People Register of Norway for 67 of the 68 patients (no match was found for a patient born in 1933). The controls were contacted by regular mail. If a positive response to participate was received, they were asked about their physical status, including any medication, and the possibility to walk $>1 \mathrm{~km}$ without any discomfort. No history of cardiovascular disease was accepted, but preventive doses of blood pressure treatment, statins, and low doses of aspirin were allowed. The study was approved by the regional committee of health and medical research ethics in Southeast Norway.

Serum antibody assays. Serum antihistidyl-t-RNA synthetase (Jo1) and anti-SSA autoantibodies were detected by automated ELISA (EliA, Phadia). Four additional anti-aaRS [anti-threonyl- (PL-7), anti-alanyl- (PL-12), anti-glycyl-, and anti-isoleucyl-] were detected by a line blot assay (Euroline Myositis kit, Euroimmune Laboratory).

Pulmonary function and ILD onset. PFT included dynamic spirometry and gas diffusing capacity. All tests were performed according to the ATS-European Respiratory Society (ERS) task force guidelines using the Vmax V6200 automated system (SensorMedics, VIASYS Respiratory Care Inc. $)^{24,25}$. Recorded variables were the FVC and the forced expiratory volume in $1 \mathrm{~s}$ (FEV1). Gas diffusing variables were the transfer factor for carbon monoxide (DLCO) and DLCO divided by alveolar volume (DLCO/VA). Values were expressed in absolute figures and as percentage of predicted normal values. Reference values were those recommended by the ERS ${ }^{26}$. Data on baseline PFT for the antisynthetase syndrome group were collected by manual chart review. Onset of ILD was defined as acute or gradual according to Tillie-Leblond, et $a l^{27}$.

Six-minute walking distance. The 6MWD was performed on a flat walking lane of $35 \mathrm{~m}$ and according to the ATS guidelines ${ }^{28}$.

Acquisition and review of CT images. Volumetric multidetector CT was performed in the supine position during breath-holding and deep inspiration with supplementary scans in the prone position when necessary. The images were reconstructed at $1-1.25 \mathrm{~mm}$ section thickness with $10-\mathrm{mm}$ intervals and at 2.5 -mm section thickness reformatted in 3 planes. Three chest radiologists blinded to the patient's condition reviewed the images in consensus and in random order. The observers evaluated the presence and extent of ILD including findings such as reticular pattern, ground-glass opacities, airspace consolidations, parenchymal bands, subpleural curvilinear lines, and traction bronchiectasis ${ }^{29}$. Reticular pattern was classified into 3 grades: (1) a fine intralobular pattern without evident cysts, (2) a pattern with predominant small cysts involving air spaces $\leq 4 \mathrm{~mm}$ in diameter, and (3) a pattern with larger cysts involving air spaces $>4 \mathrm{~mm}^{30}$. Different patterns of ILD usually coexisted, but were not specifically diagnosed. The ILD changes were reviewed by 2 different methods: (1) area measurements were done precisely by drawing a freehand region of interest on the Picture Archiving and Communication System screen to score overall extent of ILD on $10 \mathrm{CT}$ sections evenly spaced throughout the lung, and relating this to the total lung volume. The results were expressed as percent ILD of total lung volume $^{31}$; and (2) total ILD score was determined by assessing abnormalities consistent with ILD in 8 zones, 4 for each lung. The extent of involvement was evaluated independently for each zone, and each zone was assigned a score $(0-10)$ based on the percentage of lung parenchyma that showed evidence of ILD. Total ILD scores were calculated by adding up scores from the 8 lung zones for each patient, giving a maximum score of $80^{31}$.

Myositis and arthritis activity. A patient had ongoing myositis if 2 out of 3 of the following tests were positive: (1) elevated creatine kinase level $>2 \times$ upper limit of normal, (2) presence of muscle edema in muscle magnetic resonance imaging, and (3) reduced muscle strength evaluated with the manual muscle test (MMT) on 14 muscles as by Kendall, et $a l^{32}$. Ongoing arthritis was evaluated clinically by the principal investigator (HA).

Statistical analyses. SPSS version 22.0 (SPSS) was used for statistical analyses. Descriptive data were reported as mean and SD in normal distributed variables and median with range in non-normal distribution. Differences in continuous variables were tested with the Student $t$ test for normally distributed variables and the Mann-Whitney $U$ test for non-normally distributed data. The chi-square test was used to detect association between categorical variables. Correlations between FVC, DLCO, and extent ILD were detected by Pearson correlation. Estimates of the effects of various clinical manifestations on FVC\%, DLCO \%, and extent ILD\% were performed by linear regression analyses. Statistical significance was defined as $\mathrm{p}<0.05$.

\section{RESULTS}

Characteristics of the total antisynthetase syndrome cohort and the antisynthetase syndrome study cohort. By September 2011, the total antisynthetase syndrome cohort included 96 
patients; 70 were alive and 26 were dead. Except for higher age at diagnosis and lower frequency of mechanic hands in the dead, there were no differences in demographics and clinical characteristics between the living and dead patients (Supplementary Table 1, available online at jrheum.org).

All the 70 patients who were alive by September 2011 were asked to participate in our current cross-sectional comparative study. Two patients declined. Hence, the antisynthetase syndrome study cohort included 68 patients (53 with anti-Jo1 antibodies, 6 anti-PL-7, and 9 anti-PL-12). Median disease duration at inclusion was 71 months (6-362). Clinical ILD was evident in 66 out of 68 of the patients, 10 of whom had an acute onset of ILD. Seventy-seven percent had been diagnosed with myositis. Polyarthritis was diagnosed during disease course in 15 of the patients with antisynthetase syndrome ( 3 with an erosive type), and mono/oligoarthritis in 9. At the time of study inclusion, 17 patients had findings compatible with active myositis and 20 had arthritis by clinical examination.

Medical treatment of the antisynthetase syndrome study cohort. At the time of diagnosis, 66 out of 68 patients received intravenous and/or oral steroids as induction therapy. Additional treatments were given during the disease course in $>95 \%$ of the patients, mostly cyclophosphamide, azathioprine (AZA), or methotrexate (MTX). Further, 24 of the 68 patients had received at least 1 cycle with rituximab (RTX). Two patients had never been treated with immunosuppression. At study inclusion, 58 of the 68 patients were receiving maintenance therapy with oral steroids, 10 received $\geq 10 \mathrm{mg}$ of prednisone/day and 48 received $<10 \mathrm{mg}$ of prednisone/day. Eleven patients were receiving steroid monotherapy. Other drugs used for maintenance were AZA $(\mathrm{n}=27)$, mycophenolate $(\mathrm{n}=14), \operatorname{MTX}(\mathrm{n}=5)$, cyclosporine $(n=3)$, tacrolimus $(n=1)$, and RTX $(n=1)$. Six patients were not receiving any medical therapy. Overall, each patient had been treated with a median of 2 disease-modifying drugs before the ongoing treatment (range 0-8).

Pulmonary function test. Data on FVC, FEV1, and DLCO were retrievable in 67,66, and 63 patients, respectively. Four patients were not able to perform the DLCO test because of severe lung involvement. Compared with the matched controls, the antisynthetase syndrome group had mean $28 \%$, 27\%, and 53\% lower values for FVC, FEV1, and DLCO, respectively ( $p<0.001$; Figure 1). The results of FVC, FEV1, DLCO, and DLCO/VA are seen in Table 1. Smoking habits did not differ between patients (9 smokers) and controls (14 smokers; $p<0.237)$. There was no significant difference between baseline and followup PFT data in the antisynthetase syndrome group: mean FVC was 79\% at baseline and $83 \%$ at followup, mean FEV1 was $74 \%$ versus $79 \%$, and mean DLCO was $58 \%$ versus $62 \%$.

In a univariable and multivariable linear regression model, FVC\% at study inclusion was associated with disease duration and anti-SSA. The same was seen for FEV\% (data not shown), but not for DLCO\% (Supplementary Table 2, available online at jrheum.org). No correlation between $\mathrm{FVC} \%$ and MMT was found.

Six-minute walking distance. The 59 out of 68 patients who were able to perform the $6 \mathrm{MWD}$ walked a mean distance of $543 \mathrm{~m}$ (SD 113), a mean of $116 \mathrm{~m}$ shorter than the $659 \mathrm{~m}$ (SD 69) walked by the controls ( $p<0.001$; Table 1$)$. Of the 9 patients who did not perform the walking test, 5 patients had arthritis, 2 had severe lung involvement, and 2 were wheelchair bound. For 1 patient, there was no available test result. No significant difference in the 6MWD was seen between the Jo1 and non-Jo1 group (Table 1). In the antisynthetase syndrome group, the 6MWD correlated significantly with FVC $(p<0.01)$ and DLCO $(p<0.01)$, but not with ILD

Table 1. Overview of demographics and pulmonary involvement in the antisynthetase syndrome cohort and the matched controls. Values are mean (SD) unless otherwise specified.

\begin{tabular}{|c|c|c|c|c|c|c|}
\hline \multirow[t]{2}{*}{ Characteristics } & \multicolumn{3}{|c|}{ Antisynthetase Syndrome vs Controls } & \multicolumn{3}{|c|}{ Antisynthetase Syndrome Subset Analyses } \\
\hline & $\begin{array}{l}\text { Antisynthetase Syndrome, } \\
\qquad \mathrm{n}=68\end{array}$ & Controls, $\mathrm{n}=67$ & $\mathrm{p}$ & $\mathrm{Jo} 1, \mathrm{n}=53$ & $\mathrm{PL}-7 /-12, \mathrm{n}=15$ & $\mathrm{p}$ \\
\hline Disease duration, mos, median (range) & $71(6-362)$ & NA & & $76(6-362)$ & $35(7-230)$ & $<0.004$ \\
\hline Acute-onset ILD, n/N (\%) & $10 / 66(15)$ & NA & & $6 / 51(12)$ & $4 / 15(27)$ & NS \\
\hline Anti-SSA, n/N (\%) & $54 / 68(79)$ & NA & & $43(81)$ & $11(73)$ & NS \\
\hline $\mathrm{FVC}, 1$ & $2.92(1.09)$ & $3.73(0.99)$ & $<0.001$ & $2.83(1.07)$ & $3.24(1.11)$ & NS \\
\hline FEV1,1 & $2.30(0.83)$ & $2.91(0.79)$ & $<0.001$ & $2.19(0.83)$ & $2.61(0.84)$ & NS \\
\hline $\mathrm{DLCO}, \mathrm{mmol} / \mathrm{kPa} \cdot \mathrm{min}$ & $5.53(1.84)$ & $8.45(2.29)$ & $<0.001$ & $5.53(1.75)$ & $5.46(2.28)$ & NS \\
\hline $\mathrm{DLCO} / \mathrm{VA}, \mathrm{mmol} / \mathrm{kPa} \cdot \mathrm{min}$ & $1.33(0.30)$ & $1.50(0.24)$ & $<0.002$ & $1.34(0.33)$ & $1.26(0.25)$ & NS \\
\hline \multicolumn{7}{|l|}{ HRCT abnormalities } \\
\hline ILD extent, $\%$, median (range) & $20(0-73)$ & NA & & $18(3-73)$ & $26(0-70)$ & NS \\
\hline
\end{tabular}

ILD: interstitial lung disease; 6MWD: 6-min walking distance; FVC: forced vital capacity; FEV1: forced expiratory volume in $1 \mathrm{~s}$; VA: alveolar volume; HRCT: high-resolution computed tomography; NA: not available; NS: not significant.

Personal non-commercial use only. The Journal of Rheumatology Copyright @ $@ 2016$. All rights reserved 


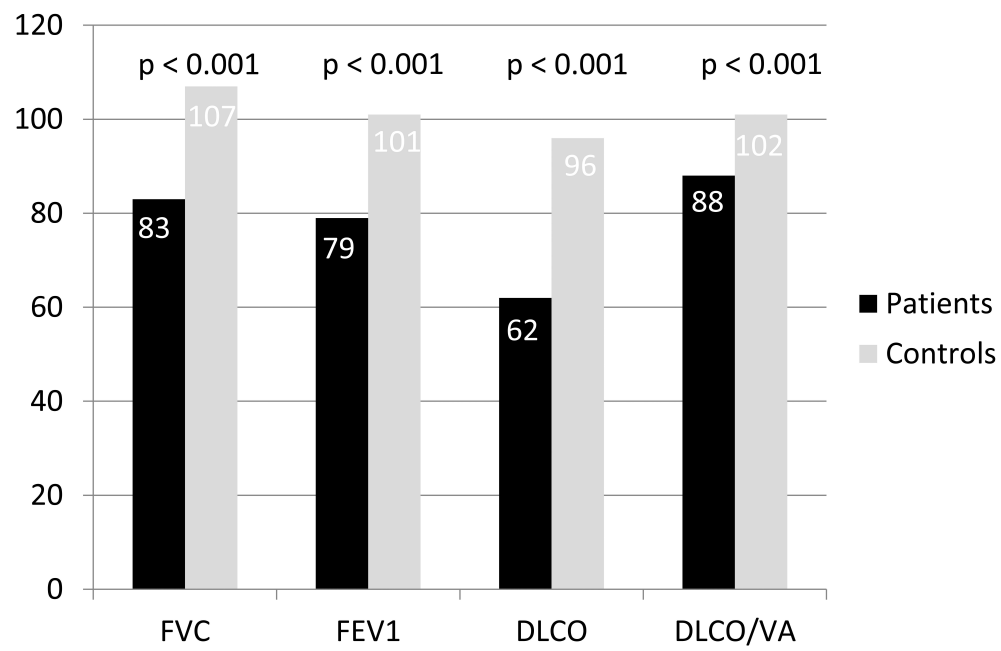

Figure 1. Comparison of PFT between the antisynthetase syndrome cohort (black stacks) and controls (grey stacks). All the PFT values are expressed as percentage of expected values. PFT: pulmonary function tests; FVC: forced vital capacity; FEV1: forced expiratory volume in $1 \mathrm{~s}$; VA: alveolar volume.

Table 2. Frequency of different HRCT patterns in the antisynthetase syndrome cohort.

\begin{tabular}{lc}
\hline HRCT Patterns & Frequency (\%) \\
\hline Ground-glass opacities & $7(10)$ \\
Opacities/infiltrates & $8(12)$ \\
Reticular pattern grade 1 & $35(52)$ \\
Reticular pattern grade 2 & $26(38)$ \\
Reticular pattern grade 3 & $14(21)$ \\
Subpleural curvilinear lines and parenchymal bands & $58(85)$ \\
Wedge-shaped & $57(84)$ \\
Emphysema & $5(7)$ \\
\hline
\end{tabular}

HRCT: high-resolution computed tomography.

extent. In the controls, 6MWD also correlated with FVC (p $<0.02)$ and DLCO $(\mathrm{p}<0.035)$.

Evaluation of CT images. Signs of ILD were identified in 66 out of 67 patients $(98.5 \%)$ by HRCT. The extent of ILD ranged from $0 \%-73 \%$, with a median value of $20 \%$ (Figure 2A). Median total ILD score was 16 (range 0-58; Table 1). The most common ILD patterns observed were subpleural curvilinear lines and parenchymal bands seen in $85 \%$ of the patients (Table 2). More than $80 \%$ of the patients displayed ILD changes distributed in a wedge-shaped pattern, predominantly in the lower part of the lungs (Figure 2B). Regression analyses showed no association between extent of ILD, anti-aaRS, anti-SSA, disease duration, or acute onset of ILD (Supplementary Table 2, available online at jrheum.org). Correlation analyses showed that ILD extent was negatively correlated with FVC\% ( $\mathrm{r}=-0.348, \mathrm{p}<0.01)$ and DLCO \% $(\mathrm{r}=-0.539, \mathrm{p}<0.01$; Figure 3$)$.

Comparison of antisynthetase syndrome subgroups. Disease duration was significantly different between the 2 subgroups: median 76 months (range 6-362) in anti-Jo1-positive patients, and 35 months (range 7-230) in the anti-Jo1-negative group ( $\mathrm{p}<0.004$; Table 1). Further, disease duration had an effect on FVC\% (p < 0.02) and FEV1\% (data not shown), but not on $\mathrm{DLCO} \%$, in a multivariable linear regression analysis that included antisynthetase syndrome subgroups, acute onset of ILD, and anti-SSA (Supplementary Table 2, available online at jrheum.org). There was a trend toward more acute onset of ILD in the non-Jo1 group (27\%) compared with the Jo1 group (12\%), and also a tendency toward more ILD extent in the non-Jo1 group, 26\% compared with $18.5 \%$ in the Jo1 group (Table 1). No significant difference between the antisynthetase syndrome subsets was found in age at diagnosis, FVC, FEV1, DLCO, DLCO/VA, or SSA antibody status (Table 1).

\section{DISCUSSION}

Comparative, quantitative data on longterm pulmonary outcome in antisynthetase syndrome are limited. Here, we show that FVC and FEV1 were reduced by $25 \%$ and DLCO by $50 \%$ in patients with antisynthetase syndrome with a median of 6 years of disease duration compared with healthy matched controls. Importantly, there was a good correlation between DLCO, FVC, and the extent of ILD by HRCT in the antisynthetase syndrome cohort.

In comparison with our large systemic sclerosis (SSc) cohort, where the PFT had a tendency to decline ${ }^{31}$, the trend in our current study was toward increased PFT at followup. This could, at least to some extent, be explained by the different responsiveness to immune therapy for the 2 entities. Notably, one-third of the patients with antisynthetase syndrome received RTX, a drug we have found to exert effects on antisynthetase syndrome-associated ILD ${ }^{33}$. However, with about the same time from first symptom to

Personal non-commercial use only. The Journal of Rheumatology Copyright $\subset$ $\subset$ 2016. All rights reserved 
A

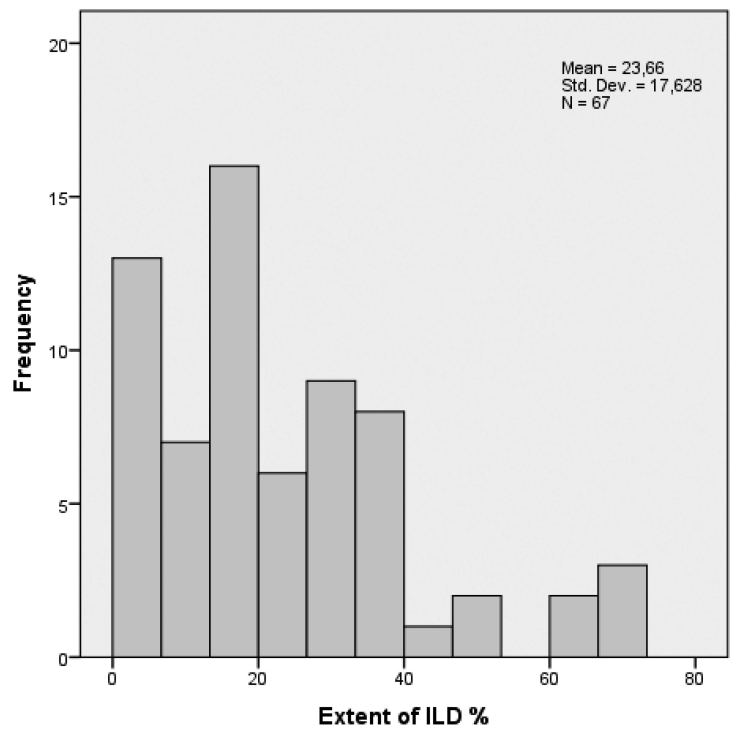

B

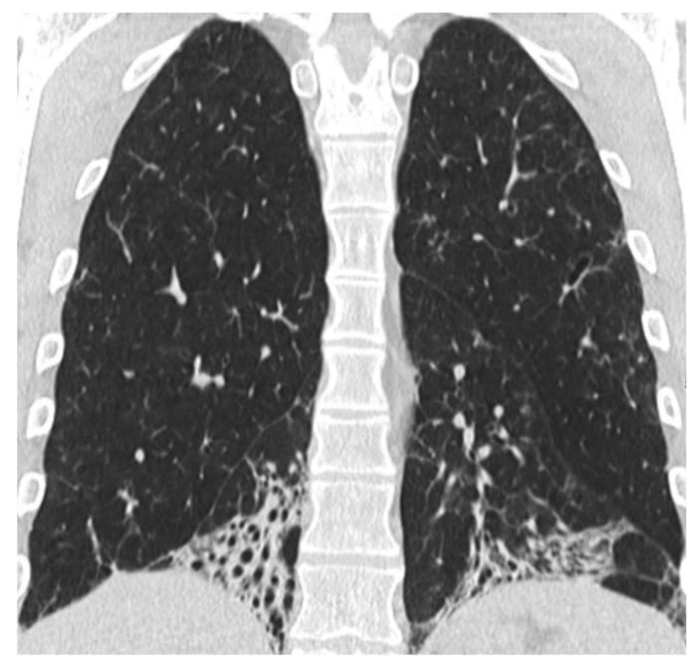

Figure 2. HRCT abnormalities in antisynthetase syndrome. A. Frequency distribution of ILD extent (in percentage of total volume of lung parenchyma) by HRCT across the antisynthetase syndrome cohort. B. HRCT image of a patient with antisynthetase syndrome with characteristic reticular pattern in a wedge-shaped distribution (inferior part of the right lung). HRCT: high-resolution computed tomography; ILD: interstitial lung disease.

A

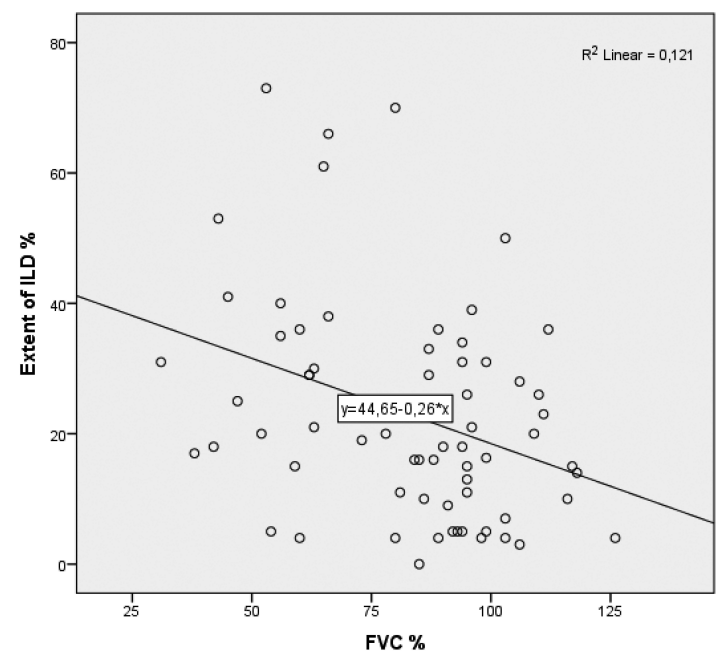

B

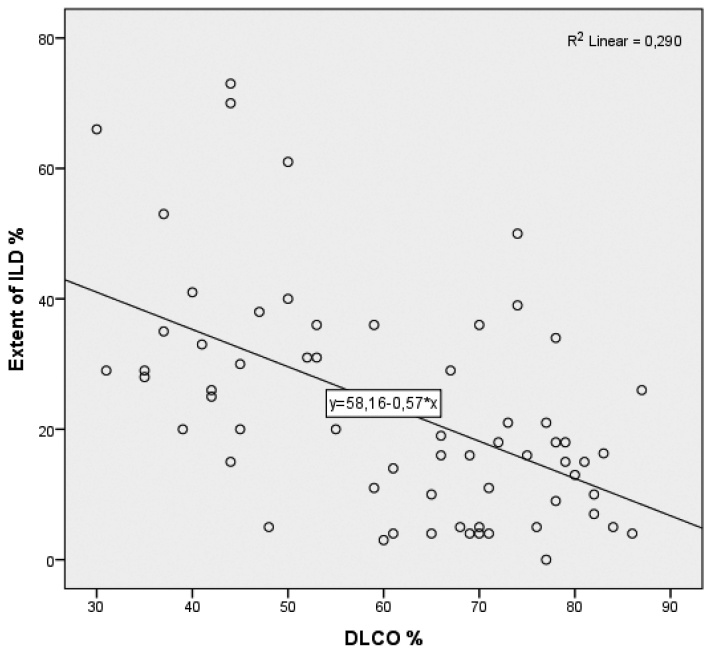

Figure 3. A. Correlation between FVC\% and ILD extent in the antisynthetase syndrome cohort. B. Correlation between DLCO \% and ILD extent in the antisynthetase syndrome cohort. ILD extent in percentage of total volume of lung parenchyma. FVC: forced vital capacity; ILD: interstitial lung disease.

baseline PFT in both cohorts, the mean baseline values of $\mathrm{FVC} \%$ and DLCO $\%$ were lower in SSc $(70 \%$ and $48 \%$, respectively) than in antisynthetase syndrome (79\% and $58 \%$ ), indicating that chronic SSc-associated ILD has a more profound effect on pulmonary function than the ILD of antisynthetase syndrome. The tendency to increased PFT at followup in our study is consistent with other studies in antisynthetase syndrome-associated ILD ${ }^{8,18}$.

Hemoglobin ( $\mathrm{Hb}$ ) could influence DLCO, but since mean
$\mathrm{Hb}$ values in the patient group $(14.3 \mathrm{~g} / \mathrm{dl}$ for men and 13.1 $\mathrm{g} / \mathrm{dl}$ for women) did not differ from standard reference values $^{24}$, we do not believe that $\mathrm{Hb}$ had any effect on DLCO in our study.

DLCO is reduced in ILD whatever the underlying cause, but can also be affected by disease processes other than ILD, for example, emphysema. In our study, only 5 of the patients with antisynthetase syndrome had signs of emphysema by HRCT. At a median of $61 \%$ of expected (range 35-84), the 
DLCO values of the 5 patients with emphysema were similar to the $62 \%$ observed in the patients with antisynthetase syndrome without emphysema, indicating that emphysema could only to a minor extent contribute to the reduction of DLCO. Another possible explanation for the reduced DLCO could be precapillary pulmonary hypertension $(\mathrm{PH})$, a feared complication in longstanding antisynthetase syndrome ${ }^{34,35,36}$. Altogether, 10 out of 68 patients had right heart catheterization during disease course, with $\mathrm{PH}$ diagnosed in 5 out of 10. Four of the 5 patients with PH had DLCO $<45 \%$ of expected (range 35-44); in the last patient with PH, DLCO was not measurable. Hence, it appears that PH contributed to some extent to the low DLCO observed.

The CTD-ILD working group of the Outcome Measures in Rheumatology suggested total ILD extent by HRCT as 1 of 2 appropriate core domains for prospective ILD studies; the other was $\mathrm{FVC}^{20}$. The myositis component can influence FVC, but in our study, there was no correlation between FVC\% and muscle strength in the patients with antisynthetase syndrome. One explanation could be that MMT is not a specific test for the respiratory muscles. Several studies describe the different CT abnormalities in antisynthetase syndrome $8,9,18,37,38,39,40$; some of them also use a scoring system combining the abnormalities with the ILD extent ${ }^{8,9,18,40}$. Here, we found that a median of $20 \%$ of the total lung parenchyma had changes consistent with ILD. Interestingly, we noticed a significant correlation between the extent of ILD and FVC, suggesting that lung capacity and radiological abnormalities are closely linked in antisynthetase syndrome-ILD. However, an even stronger correlation was found between DLCO and ILD extent, indicating that DLCO may also be a valuable outcome measure in antisynthetase syndrome-ILD.

Even though the HRCT analyses in our current study were focused on ILD extent, we also described the different types of abnormalities observed. In over $80 \%$ of the patients, a wedge-shaped distribution pattern of the ILD abnormalities was noted. The importance of this observation is not clear, but we believe that it warrants further investigation.

Interpretation of the 6MWD test in antisynthetase syndrome is difficult because the results might be influenced by arthritis and the myositis component. In our study, 30\% of the patients had ongoing myositis and/or arthritis at study inclusion. We did, however, find similar correlations between 6MWD, FVC, and DLCO in both patients and controls, indicating that this test, at least partly, reflected the functional lung capacity of the patients. There was, however, no correlation between the 6MWD and the extent of ILD. Notably, we did not measure peripheral oxygen saturation before and after the 6MWD. It is possible that the addition of this variable would have increased the information value of the 6MWD.

The antisynthetase syndrome subset analyses showed that Jo1 and non-Jo1 (i.e., PL-7 and PL-12) patients had comparable PFT, but differed numerically in the frequency of acute-onset ILD and the extent of ILD. These differences were not significant, and we speculate that the 2 main reasons for this were the shorter disease duration of the non-Jo1 subset and the low number of non-Jo1 cases. Overall, it appears that our PFT and CT data are consistent with antisynthetase syndrome subset studies with shorter time of followup ${ }^{15,18}$, and also with the recent, small-sized, longterm followup study from Tomonaga, et al in $\mathrm{Japan}^{41}$.

Our current study has potential limitations. First, the study design, with complete patient inclusion before enrollment of the controls, gave a mean 14-month lag phase between the examination of the patient and their corresponding control. Hence, the controls were 1 year older than the patients when they were tested, potentially giving a negative age effect on PFT and 6MWD performances in the controls. Second, because we did not perform detailed assessments of treatments in the antisynthetase syndrome group, we do not know whether any of the antisynthetase syndrome subset analyses were influenced by differences in treatment.

Our study, which is the first to compare a well-defined antisynthetase syndrome cohort with healthy controls, shows a significantly reduced pulmonary function in the antisynthetase syndrome group. A significant correlation between FVC/DLCO and the extent of ILD was registered, indicating that these variables are interrelated and useful as outcome measures in antisynthetase syndrome-ILD. Our study highlights the severity of pulmonary involvement in the antisynthetase syndrome and its need for effective treatment. Collaborative clinical trials are needed to assess this issue.

\section{ACKNOWLEDGMENT}

We thank our study nurse, Helga Grimstad Sørhøy, for coordinating the investigations of the controls.

\section{ONLINE SUPPLEMENT}

Supplementary data for this article are available online at jrheum.org.

\section{REFERENCES}

1. Marguerie C, Bunn CC, Beynon HL, Bernstein RM, Hughes JM, So $\mathrm{AK}$, et al. Polymyositis, pulmonary fibrosis and autoantibodies to aminoacyl-tRNA synthetase enzymes. Q J Med 1990;77:1019-38.

2. Kalluri M, Sahn SA, Oddis CV, Gharib SL, Christopher-Stine L, Danoff SK, et al. Clinical profile of anti-PL-12 autoantibody. Cohort study and review of the literature. Chest 2009;135:1550-6.

3. Yamasaki Y, Yamada H, Nozaki T, Akaogi J, Nichols C, Lyons R, et al. Unusually high frequency of autoantibodies to PL-7 associated with milder muscle disease in Japanese patients with polymyositis/dermatomyositis. Arthritis Rheum 2006;54:2004-9.

4. Mahler M, Miller FW, Fritzler MJ. Idiopathic inflammatory myopathies and the anti-synthetase syndrome: a comprehensive review. Autoimmun Rev 2014;13:367-71.

5. Cavagna L, Nuno L, Scire CA, Govoni M, Longo FJ, Franceschini F, et al. Clinical spectrum time course in anti Jo-1 positive antisynthetase syndrome: results from an international retrospective multicenter study. Medicine 2015;94:e1144.

6. Chen IJ, Jan Wu YJ, Lin CW, Fan KW, Luo SF, Ho HH, et al. Interstitial lung disease in polymyositis and dermatomyositis. Clin Rheumatol 2009;28:639-46 
7. Hallowell RW, Danoff SK. Interstitial lung disease associated with the idiopathic inflammatory myopathies and the antisynthetase syndrome: recent advances. Curr Opin Rheumatol 2014;26:684-9.

8. Marie I, Josse S, Hatron PY, Dominique S, Hachulla E, Janvresse A, et al. Interstitial lung disease in anti-Jo-1 patients with antisynthetase syndrome. Arthritis Care Res 2013;65:800-8.

9. Stanciu R, Guiguet M, Musset L, Touitou D, Beigelman C, Rigolet A, et al. Antisynthetase syndrome with anti-Jo1 antibodies in 48 patients: pulmonary involvement predicts disease-modifying antirheumatic drug use. J Rheumatol 2012;39:1835-9.

10. Hervier B, Devilliers H, Stanciu R, Meyer A, Uzunhan Y, Masseau A, et al. Hierarchical cluster and survival analyses of antisynthetase syndrome: phenotype and outcome are correlated with anti-tRNA synthetase antibody specificity. Autoimmun Rev 2012;12:210-7.

11. La Corte R, Lo Mo Naco A, Locaputo A, Dolzani F, Trotta F. In patients with antisynthetase syndrome the occurrence of anti-Ro/SSA antibodies causes a more severe interstitial lung disease. Autoimmunity 2006;39:249-53.

12. Marie I, Hatron PY, Dominique S, Cherin P, Mouthon L, Menard JF, et al. Short-term and long-term outcome of anti-Jo1-positive patients with anti-Ro52 antibody. Semin Arthritis Rheum 2012;41:890-9.

13. Váncsa A, Csípo I, Németh J, Dévényi K, Gergely L, Dankó K. Characteristics of interstitial lung disease in SS-A positive/Jo-1 positive inflammatory myopathy patients. Rheumatol Int 2009;29:989-94.

14. Aggarwal R, Cassidy E, Fertig N, Koontz DC, Lucas M, Ascherman DP, et al. Patients with non-Jo-1 anti-tRNA-synthetase autoantibodies have worse survival than Jo-1 positive patients. Ann Rheum Dis 2014;73:227-32.

15. Johnson C, Connors GR, Oaks J, Han S, Truong A, Richardson B, et al. Clinical and pathologic differences in interstitial lung disease based on antisynthetase antibody type. Respir Med 2014; 108:1542-8

16. Hamaguchi Y, Fujimoto M, Matsushita T, Kaji K, Komura K, Hasegawa M, et al. Common and distinct clinical features in adult patients with anti-aminoacyl-tRNA synthetase antibodies: heterogeneity within the syndrome. PLoS One 2013;8:e60442.

17. Hervier B, Benveniste O. Clinical heterogeneity and outcomes of antisynthetase syndrome. Curr Rheumatol Rep 2013;15:349.

18. Marie I, Josse S, Decaux O, Dominique S, Diot E, Landron C, et al. Comparison of long-term outcome between anti-Jo1- and anti-PL7/PL12 positive patients with antisynthetase syndrome. Autoimmun Rev 2012;11:739-45.

19. Debray MP, Borie R, Revel MP, Naccache JM, Khalil A, Toper C, et al. Interstitial lung disease in anti-synthetase syndrome: Initial and follow-up CT findings. Eur J Radiol 2015;84:516-23.

20. Saketkoo LA, Mittoo S, Huscher D, Khanna D, Dellaripa PF, Distler O, et al; CTD-ILD Special Interest Group. Connective tissue disease related interstitial lung diseases and idiopathic pulmonary fibrosis: provisional core sets of domains and instruments for use in clinical trials. Thorax 2014;69:428-36.

21. American Thoracic Society. Idiopathic pulmonary fibrosis: diagnosis and treatment. International consensus statement. American Thoracic Society (ATS), and the European Respiratory Society (ERS). Am J Respir Crit Care Med 2000;161:646-64.

22. Bohan A, Peter JB. Polymyositis and dermatomyositis (second of two parts). N Engl J Med 1975;292:403-7.

23. Dobloug C, Garen T, Bitter H, Stjärne J, Stenseth G, Grøvle L, et al. Prevalence and clinical characteristics of adult polymyositis and dermatomyositis; data from a large and unselected Norwegian cohort. Ann Rheum Dis 2015;74:1551-6.

24. Macintyre N, Crapo RO, Viegi G, Johnson DC, van der Grinten CP, Brusasco V, et al. Standardisation of the single-breath determination of carbon monoxide uptake in the lung. Eur Respir J 2005; 26:720-35
25. Miller MR, Hankinson J, Brusasco V, Burgos F, Casaburi R, Coates A, et al; ATS/ERS Task Force. Standardisation of spirometry. Eur Respir J 2005;26:319-38.

26. Quanjer PH, Tammeling GJ, Cotes JE, Pedersen OF, Peslin R, Yernault JC. Lung volumes and forced ventilatory flows. Report Working Party Standardization of Lung Function Tests, European Community for Steel and Coal. Official Statement of the European Respiratory Society. Eur Respir J Suppl 1993;16:5-40.

27. Tillie-Leblond I, Wislez M, Valeyre D, Crestani B, Rabbat A, Israel-Biet D, et al. Interstitial lung disease and anti-Jo-1 antibodies: difference between acute and gradual onset. Thorax 2008;63:53-9.

28. ATS Committee on Proficiency Standards for Clinical Pulmonary Function Laboratories. ATS statement: guidelines for the six-minute walk test. Am J Respir Crit Care Med 2002;166:111-7.

29. Hansell DM, Bankier AA, MacMahon H, McLoud TC, Müller NL, Remy J. Fleischner Society: glossary of terms for thoracic imaging. Radiology 2008;246:697-722.

30. Gunnarsson R, Aaløkken TM, Molberg Ø, Lund MB, Mynarek GK, Lexberg AS, et al. Prevalence and severity of interstitial lung disease in mixed connective tissue disease: a nationwide, cross-sectional study. Ann Rheum Dis 2012;71:1966-72.

31. Hoffmann-Vold AM, Aaløkken TM, Lund MB, Garen T, Midtvedt $\varnothing$, Brunborg C, et al. Predictive value of serial high-resolution computed tomography analyses and concurrent lung function tests in systemic sclerosis. Arthritis Rheumatol 2015;67:2205-12.

32. Kendall FP, McCreary EK, Provance PG. Muscles: testing and function. Baltimore: Williams \& Wilkins; 1993.

33. Andersson H, Sem M, Lund MB, Aaløkken TM, Günther A, Walle-Hansen R, et al. Long-term experience with rituximab in anti-synthetase syndrome-related interstitial lung disease. Rheumatology 2015;54:1420-8.

34. Handa T, Nagai S, Kawabata D, Nagao T, Takemura M, Kitaichi M, et al. Long-term clinical course of a patient with anti PL-12 antibody accompanied by interstitial pneumonia and severe pulmonary hypertension. Intern Med 2005;44:319-25.

35. Hervier B, Meyer A, Dieval C, Uzunhan Y, Devilliers H, Launay D, et al. Pulmonary hypertension in antisynthetase syndrome: prevalence, aetiology and survival. Eur Respir J 2013;42:1271-82.

36. Taniguchi $\mathrm{Y}$, Horino $\mathrm{T}$, Kato $\mathrm{T}$, Terada $\mathrm{Y}$. Acute pulmonary arterial hypertension associated with anti-synthetase syndrome. Scand J Rheumatol 2010:39:179-80.

37. Ingegnoli $\mathrm{F}$, Lubatti $\mathrm{C}$, Ingegnoli $\mathrm{A}$, Boracchi $\mathrm{P}$, Zeni $\mathrm{S}$, Meroni PL. Interstitial lung disease outcomes by high-resolution computed tomography (HRCT) in Anti-Jo1 antibody-positive polymyositis patients: a single centre study and review of the literature. Autoimmun Rev 2012;11:335-40.

38. Koreeda Y, Higashimoto I, Yamamoto M, Takahashi M, Kaji K, Fujimoto M, et al. Clinical and pathological findings of interstitial lung disease patients with anti-aminoacyl-tRNA synthetase autoantibodies. Intern Med 2010;49:361-9.

39. Richards TJ, Eggebeen A, Gibson K, Yousem S, Fuhrman C, Gochuico BR, et al. Characterization and peripheral blood biomarker assessment of anti-Jo-1 antibody-positive interstitial lung disease. Arthritis Rheum 2009;60:2183-92.

40. Cavagna L, Caporali R, Abdi-Ali L, Dore R, Meloni F, Montecucco C. Cyclosporine in anti-Jo1-positive patients with corticosteroid-refractory interstitial lung disease. J Rheumatol 2013;40:484-92.

41. Tomonaga M, Sakamoto N, Ishimatsu Y, Kakugawa T, Harada T, Nakashima S, et al. Comparison of pulmonary involvement between patients expressing anti-PL-7 and anti-Jo-1 antibodies. Lung 2015;193:79-83.

Personal non-commercial use only. The Journal of Rheumatology Copyright @ 2016. All rights reserved. 